\title{
Enhanced Recovery After Surgery Impact on the Systemic Inflammatory Response of Patients Following Gynecological Oncology Surgery: A Prospective Randomized Study
}

\author{
Jin Peng (1D' \\ Ruiying Dong' \\ Jianfen Jiao $\mathbb{I D}^{\prime}$ \\ Min Liu' \\ Xi Zhang' \\ Hualei Bu' \\ Ping Dong $\mathbb{D}^{2}$ \\ Shasha Zhao (D) ${ }^{3}$ \\ Naidong Xing ${ }^{4}$ \\ Shuai Feng 5 \\ Xingsheng Yang $\mathbb{B}^{1}$ \\ Beihua Kong' \\ 'Department of Obstetrics and \\ Gynecology, Qilu hospital, Cheeloo \\ College of Medicine, Shandong \\ University, Jinan, People's Republic of \\ China; ' ${ }^{2}$ Department of Anesthesiology, \\ Qilu Hospital, Shandong University, Jinan, \\ People's Republic of China; ${ }^{3}$ Department \\ of Clinical Nutrition, Qilu Hospital, \\ Shandong University, Jinan, People's \\ Republic of China; ${ }^{4}$ Department of \\ Urology, Qilu Hospital, Shandong \\ University, Jinan, People's Republic of \\ China; ${ }^{5}$ Shandong Cancer Hospital and \\ Institute, Shandong First Medical \\ University and Shandong Academy of \\ Medical Sciences, Jinan, People's Republic \\ of China
}

Objective: Enhanced recovery after surgery (ERAS) protocol has widely gained acceptance in gynecological surgery. Its safety and efficacy should be evaluated fully via well-designed, randomized, control trials. The main objective of our study is to compare the ERAS protocol with the conventional perioperative care program after gynecological oncology. Furthermore, the secondary objectives of our study are the identification of markers that allow us to evaluate the effectiveness of the application of ERAS elements in the modulation of the body's response to surgical stress.

Methods: Patients with gynecological tumors indicated for surgery were randomly assigned to either the ERAS group or the conventional group. The ERAS protocol included short fasting time, fluid restriction, early oral feeding, reduced opioid consumption and immediate mobilization after surgery. The primary endpoint was the reduction of hospital stay in the ERAS group. The day of first flatus, postoperative nausea and vomiting (PONV), maximum pain score by the visual analogue scale (VAS) and complication, readmission rate, reoperation rate, postoperative mortality, total hospital cost and systemic inflammatory response (SIR) were secondary endpoints.

Results: A total of 130 patients in gynecological tumor surgery were enrolled (ERAS $=65$, conventional $=65$ ). The ERAS group had faster bowel function recovery, significantly less pain, less PONV, shorter hospital stay, and less total hospital costs. SIR markers were estimated and screened out that postoperative platelet, neutrophil-lymphocyte-ratio (NLR) and plateletlymphocyte-ratio (PLR) were significantly lower in ERAS groups compared to conventional groups.

Conclusion: The implementation of ERAS protocol is safe and enhances postoperative recovery after gynecological oncology surgery. We firstly reveal the beneficial effect of ERAS protocols on the alleviation of postoperative SIR, which is a reflection of the magnitude of surgical trauma. Postoperative platelet, NLR or PLR could be the novel and inexpensive markers to assess how ERAS protocols modulate gynecological oncology surgery.

Trial Registration: The trial was registered in ClinicalTrials.gov (NCT03629626).

Keywords: ERAS, enhanced recovery after surgery, systemic inflammatory response, gynecological oncology surgery, NLR, neutrophil-lymphocyte-ratio, PLR, plateletlymphocyte-ratio

\section{Introduction}

Correspondence: Xingsheng Yang Department of Obstetrics and Gynecology, Qilu Hospital, Shandong University, No. 107 West Wenhua Road, Jinan, 25000I, People's Republic of China Email xingshengyang@sdu.edu.cn
Enhanced recovery after surgery (ERAS), which is known as fast-track surgery, is a multimodal, multidisciplinary and evidence-based approach to improving perioperative care of surgical patients to enhance the quality of recovery after surgery by 
adopting a series of optimized protocols. Initial studies into enhanced recovery were mainly performed in patients undergoing colorectal surgery, but ERAS is now used in many different surgical specialties and procedures. There are consensus guidelines from the ERAS society for patients undergoing gastrointestinal surgery, urological surgery, colorectal surgery, hepatobiliary, pancreatic surgery and gynecological surgery. ${ }^{1-7}$ These guidelines have resulted in the widespread acceptance of the ERAS principles. ERAS protocols for diverse surgery types have successfully improved patient outcomes, including decreased postoperative nausea and vomiting (PONV), and decreased length of hospital stay through implementation of several evidence-based procedures, including a preoperative counseling; carbohydrate drinks 2 hours before operation and oral feeding; a standardized approach in anesthetic management and reduced opioid consumption; a restriction of tubes and catheters; early mobilization. $^{8-13}$ There is a plethora of data supporting ERAS pathways for other types of surgery, while there are moderate pieces of evidence on the application of ERAS principles to gynecological tumors surgery. ${ }^{14} \mathrm{We}$ evaluated the application of a multimodal enhanced recovery program and compared it with the conventional perioperative care for gynecological tumors surgery in the context of a prospective randomized control trial.

Since it is known that the main objective of ERAS procedures is the modulation of the systemic inflammatory response (SIR), we would like to identify some markers that allow to evaluate the effects of ERAS on the body's reaction to surgery. The trauma of surgery leads to metabolic, neuroendocrine, and immune response disorder, while the aims of ERAS are to promote physiological stability and wound healing. ${ }^{15}$ Indeed, it has been reported that concentrations of circulating acute phase proteins and cytokines are associated with the magnitude of the stress response, that is the SIR to surgery. ${ }^{16}$ The SIR involves changes in relative levels of circulating white blood cells (WBCs), and neutrophilia is accompanied by relative lymphocytopenia. ${ }^{17}$ SIR markers, such as neutrophillymphocyte-ratio (NLR), platelet-lymphocyte-ratio (PLR), and monocyte-lymphocyte-ratio (MLR), are perhaps the most clinically useful. ${ }^{18}$ Otherwise, high NLR or PLR is associated with an adverse overall survival in many solid tumors. ${ }^{19-25}$ The NLR integrates information on both the innate and adaptive compartments of the immunity and represents a reliable measure of the inflammatory burden. Furthermore, previous studies revealed relationships between the NLR and clinical outcome in patients with acute cerebral hemorrhage. ${ }^{26}$ It is known that ERAS protocols aim to reduce the systemic inflammatory response to surgery. The aim of the study is to compare the ERAS protocol with the conventional perioperative care program, and the identification of beneficial markers of the SIR for ERAS following gynecological oncology surgery.

\section{Methods}

\section{Study Design}

Patients between the age of 18-70 years, who were diagnosed with cervical tumors, uterine tumors or ovarian tumors, were eligible for enrollment. Exclusion criteria had a history of constipation and severe comorbidity, including patients with American Society of Anesthesiologists risk $\geq 4$, severe organ dysfunction or failure, a comorbidity-polypharmacy score $\geq 22 .^{27}$ A formal written consent was received from each patient after a thorough discussion. The study was approved by the institutional research board committee of the institution. The study was conducted in accordance with the Declaration of Helsinki. This study was designed as a prospective, randomized control trial with a follow-up period of 6 months. Patients were randomly assigned using block randomization on a $1: 1$ basis to either the ERAS group or the conventional group. After patients were enrolled and consented, research assistants randomized the patient into one of the two groups. It was registered under the code (NCT03629626) in ClinicalTrials.gov. The work has been reported in line with the STROCSS criteria. $^{28}$

\section{Enhanced Recovery After Surgery (ERAS) Pathways for Gynecologic Oncology Surgery}

The perioperative protocol for the two groups is summarized in Table 1. Before admission, the ERAS patients were given comprehensive preoperative education. ERAS patients were also allowed to fast up to 6 hours before surgery and intake of an oral carbohydrate solution until 2 hours before surgery. The ERAS principles of maintenance of euvolemia and normothermia were emphasized in the perioperative period. Intravenous fluid administration was restricted to a minimal amount, about 4-5 liter on the day of surgery and 1-2 liter on postoperative day (POD) 1-2, and 
Table I Protocols of ERAS Group and Conventional Group After Surgery

\begin{tabular}{|c|c|c|}
\hline Groups & ERAS & Conventional \\
\hline Before admission & Preoperative education operative risk assessment & Operative risk assessment \\
\hline Preoperative & $\begin{array}{l}\text { No bowel preparation } \\
\text { Fasting up to } 6 \mathrm{~h} \text { before surgery } \\
\text { Oral carbohydrate solution ( } 500 \mathrm{~mL} \text {, Carbohydrate } 2.5 \% \text { ) up to } 2 \text { hours before surgery }\end{array}$ & $\begin{array}{l}\text { Bowel preparation } \\
\text { Midnight fasting }\end{array}$ \\
\hline Day of surgery & $\begin{array}{l}\text { Insertion of Foley catheter } \\
\text { Antiembolic stockings } \\
\text { Fluid restriction ( } 4-5 \mathrm{~L}) \\
\text { Multimodal analgesia including use of short acting anesthetic agent remifentanil, injection with } \\
\text { bupivacaine in transabdominal surgery after incision closure } \\
\text { Continue and encourage ambulation } \\
\text { Sip of water } 2 \text { hours later after surgery }\end{array}$ & $\begin{array}{l}\text { Insertion of Foley catheter } \\
\text { Antiembolic stockings } \\
\text { Fluid (5-6L) }\end{array}$ \\
\hline Postoperative & $\begin{array}{l}\text { LMWH injection and antiembolic stockings } \\
\text { Foley removal as early as possible } \\
\text { Drain removal as early as possible } \\
\text { Continue and encourage ambulation } \\
\text { Fluid restriction (I-2 L) } \\
\text { NSAIDs Flurbiprofen Axetil } 50 \mathrm{mg} \text { iv bid for } 3 \text { days for analgesia } \\
\text { Semifluid diet in PODI } \\
\text { Soft blended diet in POD2 }\end{array}$ & $\begin{array}{l}\text { LMWH injection and } \\
\text { antiembolic stockings } \\
\text { Ambulation at morning } \\
\text { Fluid (2L-3L) } \\
\text { IV PCA } \\
\text { Semifluid diet after first } \\
\text { flatus }\end{array}$ \\
\hline
\end{tabular}

Abbreviations: LMWH, low molecular weight heparin; POD, postoperative day; IV PCA, intravenous patient-controlled analgesia; iv: intravenous.

stopped earlier for the ERAS group. Patients in ERAS group started oral feeding earlier than the patients of the conventional group, and patients in ERAS group were encouraged to drink sip of water 2 hours later after surgery and were administered semifluid diet on POD1 and a soft blended diet on POD2. Meanwhile, ambulation was encouraged starting on the day of surgery for the ERAS group. Patients in ERAS groups were given NSAIDS (Flurbiprofen Axetil 50mg intravenous bid for 3 days) on the surgery day and POD 1-2. The conventional group employed intravenous patient-controlled analgesia (IV PCA) mostly composing of opioid analgesics, such as fentanyl and morphine. If the Foley and drain is necessary for patients, Foley and drain removal is recommended for ERAS group patients as early as possible. The primary endpoint was the reduction of hospital stay in the ERAS group. Secondary outcomes included the day of first flatus, postoperative nausea and vomiting (PONV), maximum pain score by the visual analogue scale (VAS), complication, readmission rate, reoperation rate, postoperative mortality, total hospital cost and the SIR to surgery.

\section{Statistical Analysis}

Given that studies previously have showed that postoperative hospital stay was $6.5 \pm 3.5$ days for the ERAS group and $10.7 \pm 11.4$ days for the conventional group. $^{29}$ Allowing $5 \%$ of type I error with $80 \%$ power and a followup loss of $5 \%$, a total of 130 patients were required to evaluate surgical procedures and perioperative care.

Statistical analysis data were collected, and medical records were reviewed for medical data and complications. Statistical analyses were performed using SPSS 25.0 and Graph Pad Prism. Univariate analysis was used to compare baseline patient and operative characteristics between the two cohorts. For continuous variables, the $t$ test or the Mann-Whitney $U$-test was used, and for categorical variables, $t$ test or the Fisher's exact test was performed. P < 0.05 was considered statistically significant for all statistical comparisons.

\section{Results}

\section{Characters of Patients}

A total of 135 patients who underwent gynecological oncology surgery from September 2018 to September 2019 were 


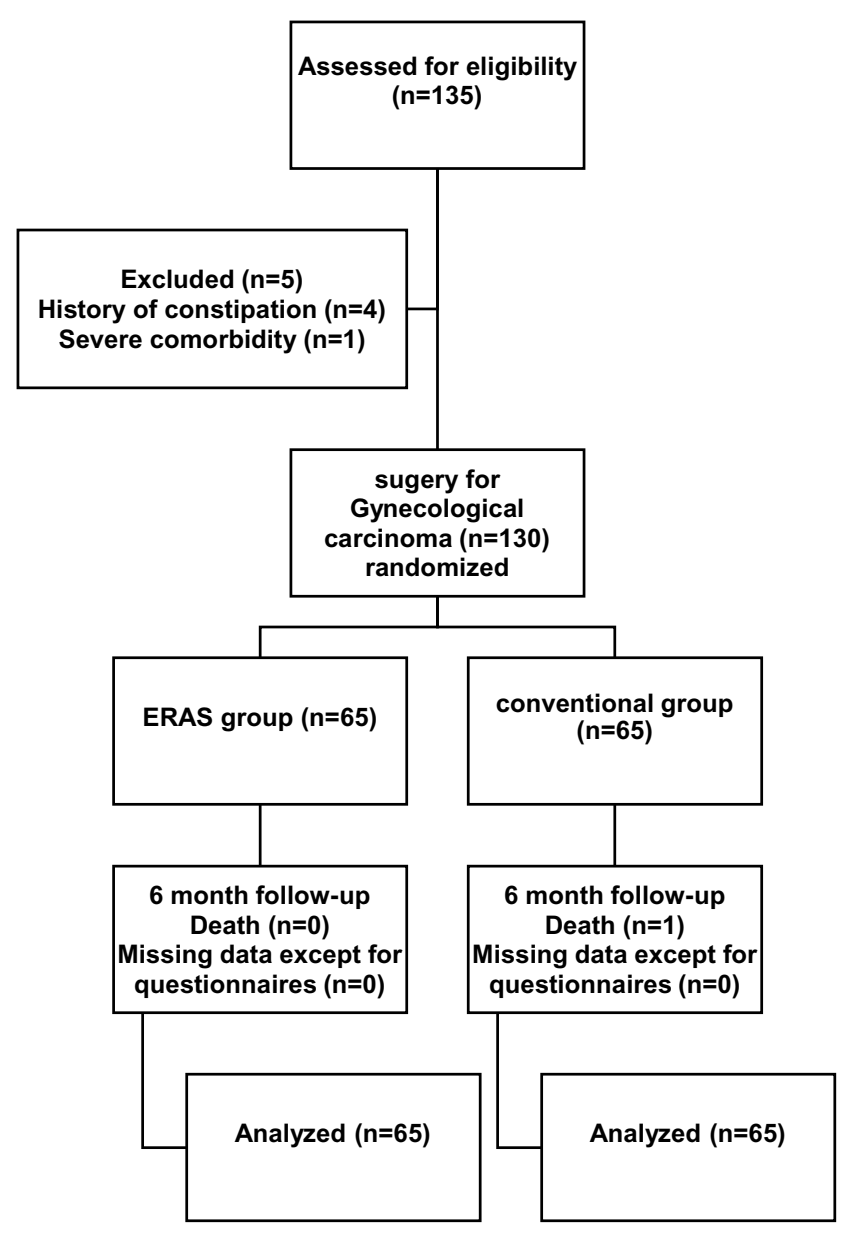

Figure I CONSORT diagram.

enrolled. Among them, 5 patients were excluded because of constipation and severe comorbidity, and finally 65 patients were assigned to the ERAS group, and 65 patients were assigned to the conventional group (Figure 1). All patients were followed up to 6 months after the operation. Patient demographics characteristics showed no statistical difference including age, BMI, diabetes rate and hypertension rate, demonstrating well randomization between the two groups (Table 2). Mean operative time was $128.4 \pm 40.74$ minutes in the ERAS group and $131.2 \pm 37.18$ minutes in the conventional group $(p=0.679$, Table 2$)$. There was no case of a conversion from a laparoscopic procedure to an open procedure. No significant differences were found in either laparoscopy rate or postoperative diagnosis data between the two groups $(p=0.249 ; p=0.235$, Table 2$)$. The dates of first sips of water, semifluid diet, and softblended diet were all significantly faster $(p=0.001$, Table 2 ), intravenous fluid intake was significantly less and stopped earlier in ERAS groups $(\mathrm{p}<0.05$, Table 2$)$, while urinary catheter and pelvic drain duration time significantly reduced in ERAS groups in accordance with the given protocol $(\mathrm{p}=0.001$, Table 2$)$.

\section{Clinical Outcome}

As we expected, the day of first flatus was faster in the ERAS group compared with the conventional group $(1.72 \pm 0.69 \mathrm{vs}$ $2.46 \pm 0.77$ days, respectively, $p=0.001$, Table 2). Despite a significant reduction in opioid, pain scores were unchanged in the women in ERAS group compared with women in the conventional group on operative day $(p=0.271$, Figure $2 A)$. Maximum pain score derived using the VAS scale was significantly higher in the conventional group from POD 1 to POD 3 ( $p=0.001$, Figure 2A). In terms of PONV, women in conventional group experienced significantly more PONV on the day of surgery after return to the ward $(59.9 \%$ in conventional group compared with $27 \%$ in ERAS group, $p=0.034$, Figure 2B). Less nausea and vomiting were still observed in the enhanced recovery group on POD 1 (26.2\% in ERAS group vs $52.3 \%$ in conventional group, $p=0.039$, Figure $2 \mathrm{~B}$ ).

Patients obviously benefit from ERAS protocols; furthermore, thirty-day rates of complications and reoperation rates do not differ between the two groups (Table 2). In the ERAS group, five patients had ileus complications. In the conventional group, there were two wound complications and three ileus. The postoperative mortality for six months and readmission rate after discharge were not significantly different between the two groups, in addition, no difference was found in subgroup analysis for cervical, uterine and ovarian cancers (Table 2). Unsurprisingly, the postoperative hospital stay was $6.91 \pm 2.35$ days in the ERAS group compared with 10.38 \pm 3.03 days in the conventional group $(p=0.0001$, Table 3 ). Introduction of enhanced recovery resulted in a 4-day reduction in the total length of stay compared with the conventional group $(\mathrm{p}=0.0001$, Table 3$)$. The reduction in the length of stay was accompanied by total hospital cost savings of more than $4500 \mathrm{RMB}$ per patient $(\mathrm{p}=0.0001$, Table 3$)$.

\section{The Modulation of ERAS Pathway on Systemic Inflammatory Response (SIR)}

Finally, we would like to demonstrate the modulation of ERAS pathway on SIR. Preoperative characteristics on the basis of peripheral blood components, including WBCs, neutrophils, lymphocytes, monocytes and platelets counts, show no difference between the ERAS group and the conventional group (Figure 3A-E). Likewise, there was no significant difference in evaluated postoperative peripheral 
Table 2 Patient Characteristics and Postoperative Course

\begin{tabular}{|c|c|c|c|}
\hline & ERAS $(n=65)$ & Conventional $(n=65)$ & $P$ value \\
\hline \multicolumn{4}{|l|}{ Demographics } \\
\hline Age & $47.46 \pm 12.90$ & $43.02 \pm 13.28$ & 0.055 \\
\hline BMI (kg/m2) & $24.63 \pm 3.95$ & $23.77 \pm 3.06$ & 0.166 \\
\hline Diabetes & $6(9.23)$ & $4(6.15)$ & \\
\hline Hypertension & $\mathrm{II}(16.9)$ & $7(10.8)$ & \\
\hline \multicolumn{4}{|l|}{ Operative data } \\
\hline Operative time (min) & $128.40 \pm 40.74$ & $131.20 \pm 37.18$ & 0.679 \\
\hline Laparoscopy cases & $28(43.08)$ & $26(40)$ & 0.249 \\
\hline Postoperative diagnosis & & & 0.235 \\
\hline Cervix cancer & $3 I(47.69)$ & $36(55.39)$ & \\
\hline Uterine cancer & $25(38.46)$ & $\mid 4(2 \mid .54)$ & \\
\hline Ovarian cancer & $6(9.24)$ & $10(15.38)$ & \\
\hline OSCST & $\mathrm{I}(\mathrm{I} .54)$ & $3(4.62)$ & \\
\hline Sarcoma & $2(3.07)$ & $2(3.07)$ & \\
\hline \multicolumn{4}{|l|}{ Postoperative course } \\
\hline Fluid by iv in day of surgery $(\mathrm{mL})$ & $4373.56 \pm 455.91$ & $5168.19 \pm 352.92$ & 0.021 \\
\hline Fluid by iv in PODI (mL) & $|602.3| \pm 249.03$ & $2406.25 \pm 428.95$ & 0.001 \\
\hline Fluid by iv in POD2(mL) & $592.91 \pm 256.19$ & $2023.68 \pm 427.32$ & 0.001 \\
\hline Fluid by iv in POD3 $(\mathrm{mL})$ & 0 & $529.38 \pm 209.12$ & 0.001 \\
\hline Sips of water (hours) & $12.16 \pm 2.25$ & $26.14 \pm 5.95$ & 0.001 \\
\hline Semifluid diet (hours) & $22.96 \pm 3.29$ & $69.54 \pm 12.96$ & 0.001 \\
\hline Soft blended diet (hours) & $48.25 \pm 10.16$ & $98.91 \pm 19.02$ & 0.001 \\
\hline First flatus (days) & $1.72 \pm 0.69$ & $2.46 \pm 0.77$ & 0.001 \\
\hline Urinary catheter duration (days) & $5.29 \pm 4.27$ & $10.77 \pm 4.78$ & 0.001 \\
\hline Pelvic drain duration (days) & $4.55 \pm 2.54$ & $6.52 \pm 3.03$ & 0.001 \\
\hline Complications & & & 0.965 \\
\hline lleus & $5(7.69)$ & $3(4.62)$ & \\
\hline Wound infection & $0(0)$ & $2(3.08)$ & \\
\hline Re-operation (\%) & $0(0)$ & $0(0)$ & 0.998 \\
\hline \multicolumn{4}{|l|}{ Postoperative mortality (\%) } \\
\hline Cervical cancer & $0(0)$ & $0(0)$ & 0.996 \\
\hline Uterine cancer & $0(0)$ & $0(0)$ & 0.998 \\
\hline Ovarian cancer & $0(0)$ & $I(10)$ & 0.689 \\
\hline Readmission (\%) & $5(7.69)$ & $6(9.23)$ & 0.469 \\
\hline
\end{tabular}

Notes: Data are $\mathrm{n}(\%)$ or mean \pm standard deviation unless otherwise specified; iv: intravenous.

Abbreviations: OSCST, ovarian sex cord stromal tumor; POD, postoperative day.

blood components, except for platelets between the two groups (Figure 3A-E). The difference between preoperative and postoperative peripheral blood components was calculated and compared, respectively, the difference of WBC, neutrophils, lymphocytes, and monocytes was not significantly different, except for the difference of platelets between two groups (Figure 3F-J). No significant difference was observed in preoperative NLR, PLR, or MLR between the two groups (Figure 4A-C). It is interesting that postoperative NLR and PLR, but not MLR, in enhanced recovery pathway patients are significantly lower compared with the conventional group (Figure 4D-F). Similarly, the difference between preoperative and postoperative NLR, PLR, but not MLR, was not significantly different between the two groups (Figure 4G-I).

\section{Discussion}

Our study revealed that patients who adhered to the enhanced recovery after the gynecological oncology surgery protocol had 4 days earlier discharge and remarkable 

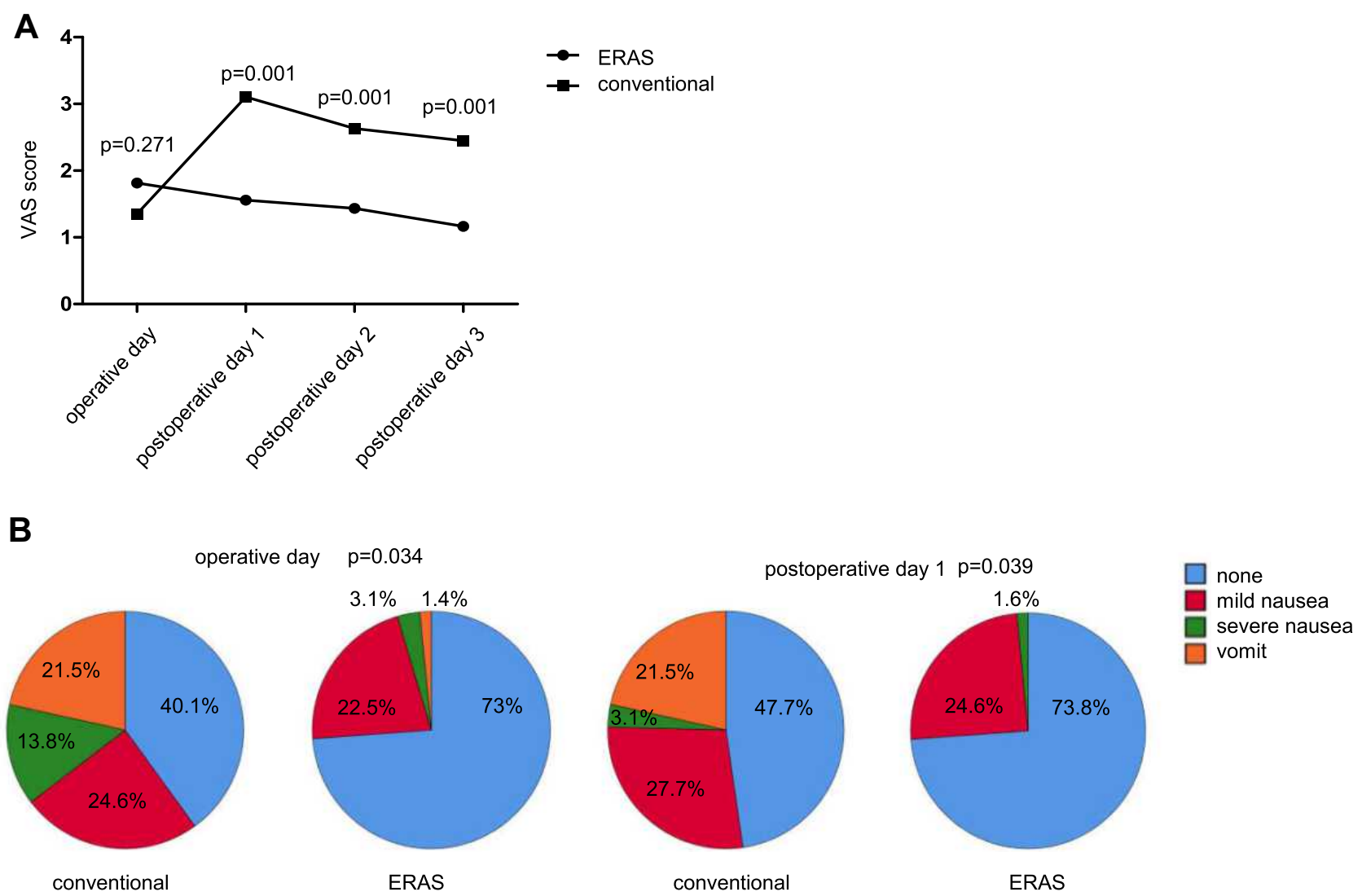

Figure 2 Postoperative results. (A) Maximum pain score assessed by the visual analogue scale (VAS); (B) postoperative nausea and vomiting (PONV) rate after surgery according to operative day and postoperative days.

economic benefit. The main reason for this difference can be attributed to faster recovery of bowel function, effective pain and PONV management using the ERAS protocol.

First, time for the first flatus has often been used as a simple way to predict bowel recovery time and prevent ileus. Because the time to first flatus was faster in the ERAS group, it can be implied that the ERAS protocol stimulated faster bowel function recovery. A proportion of patients who undergo bowel preparation with purgative laxatives and who are subject to conventional preoperative fasting protocols are subject to dehydration, electrolyte imbalance and insulin resistance. Avoidance of bowel preparation, ERAS pathway allows the use of preoperative oral carbohydrate loading and free access to fluids until 2 hours before surgery. Furthermore, there is increasing evidence that excess administration of salt solutions can delay the return of normal gastrointestinal function and can be associated with increased postoperative complications and prolonged hospital stay. ${ }^{30}$ Consistent with the fast-track surgery for colon cancer, intravenous infusion was not necessary to maintain fluid balance; thus, postoperative hospital stay was significantly shortened. ${ }^{31,32}$ Clearly, there must be a balance between achieving adequate tissue perfusion versus overloading the patient with sodium and water. Alternatively, the earlier oral intake of liquid or food can avoid excessive intravenous fluid administration, which can lead to faster bowel function recovery without increasing the incidence of fistulas. Collectively, our data are good evidence to support the safety of allowing access to fluids up to 2 hours before

Table 3 Recovery Time and Postoperative Hospital Stay

\begin{tabular}{|l|l|l|l|}
\hline & ERAS & Conventional & P value \\
\hline Postoperative hospital stay (days) & $6.91 \pm 2.35$ & $10.38 \pm 3.03$ & 0.0001 \\
Total length of hospital stay (days) & $10.69 \pm 2.95$ & $14.06 \pm 3.55$ & 0.0001 \\
Total hospital cost & $37,805.94 \pm 9828.50$ & $42,336.42 \pm 8802.89$ & 0.0001 \\
\hline
\end{tabular}



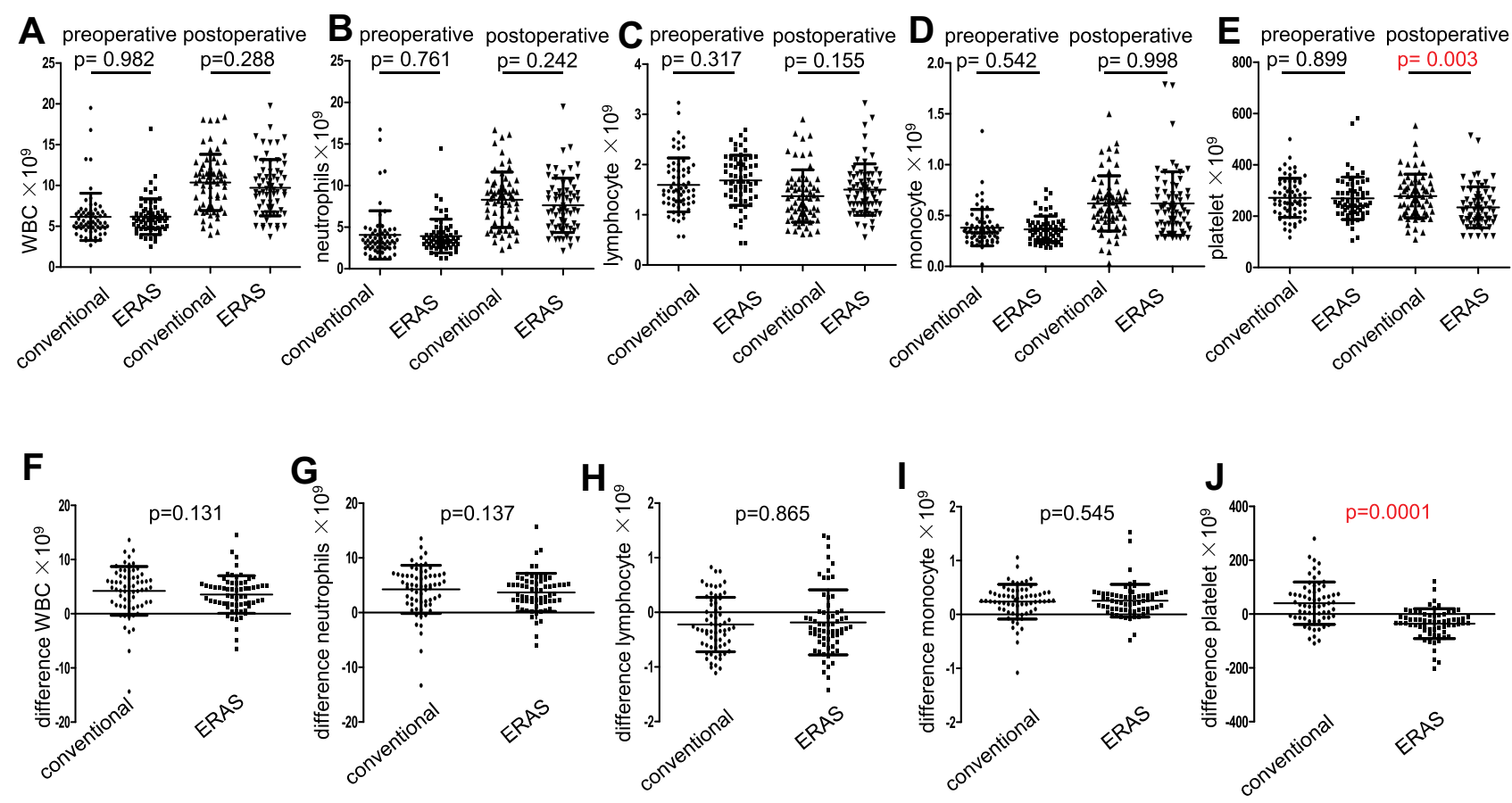

Figure 3 Comparison of patients with ERAS protocols and patients with conventional protocols, in terms of preoperative or postoperative blood subtypes or the difference between preoperative and postoperative blood subtypes. Mean of WBC counts (A), neutrophil counts (B), lymphocyte counts (C), monocyte counts (D), platelet counts $(\mathbf{E})$; difference of WBC counts $(\mathbf{F})$, neutrophil counts $(\mathbf{G})$, lymphocyte counts $(\mathbf{H})$, monocyte counts $(\mathbf{I})$ and platelet counts $(\mathbf{J})$.

gynecological oncology surgery and commence oral fluid 2 hours after surgery, due to similar postoperative complication and reoperation rate between the ERAS group and the conventional group. Although ERAS protocols show significantly less complication rates in some studies of gynecology surgery, ${ }^{30}$ our data do not show significant difference between two groups.

On the other hand, our results are consistent with prior previous studies that introduce opioid-sparing interventions, avoiding opioid-related side effects, which are not relevant to a reduction in the highest pain score. Patientcontrolled analgesia (PCA) using intravenous opioids has less beneficial physiological effects on surgical stress responses. According to our data, it is possible to achieve almost the same pain scores with NSAIDs on the surgery day after gynecological oncology surgery. Maximum pain score was significantly higher in the conventional group after gynecologic oncology surgery, which may be due to that NSAIDs were given as a baseline analgesic throughout the postoperative course in ERAS group, while the PCA using intravenous opioids is running 24 hours after surgery in the conventional group. Poor pain control postoperatively has also been associated with worse quality of life outcomes, including impaired sleep and physical function in the postoperative period and is one of the most common reasons for post-surgical hospital readmission, which can contribute to health care costs. ${ }^{31}$ Meanwhile, the locoregional analgesia was performed through injection of bupivacaine in transabdominal surgery after incision closure in ERAS group, which reduced the postoperative pain still further. Collectively, our data suggest that there is a significant improvement in pain control with ERAS implementation. Therefore, it is not surprising that our ERAS patients through the application of multimodal analgesia had a lower incidence of PONV when compared to the conventional group, due to reduction of opioid use, as well as maximizing the patient's likelihood of successful discharge. Otherwise, the true incidence of PONV of the conventional group would be underestimated if antiemetics were co-administered with opioids to prevent nausea postoperatively in our study.

Except the clinical outcome beneficial from ERAS protocol, this study also highlights that the traditional metrics including length of stay may not be sufficient to evaluate the success of an ERAS pathway. We believe there must be a shift in how we evaluate the success of an ERAS protocol. Instead of focusing on length of stay, we focus on modulation of ERAS protocols for SIR to surgery. We assumed that these elements of ERAS protocols would have the ability to reduce the SIR to surgery. 

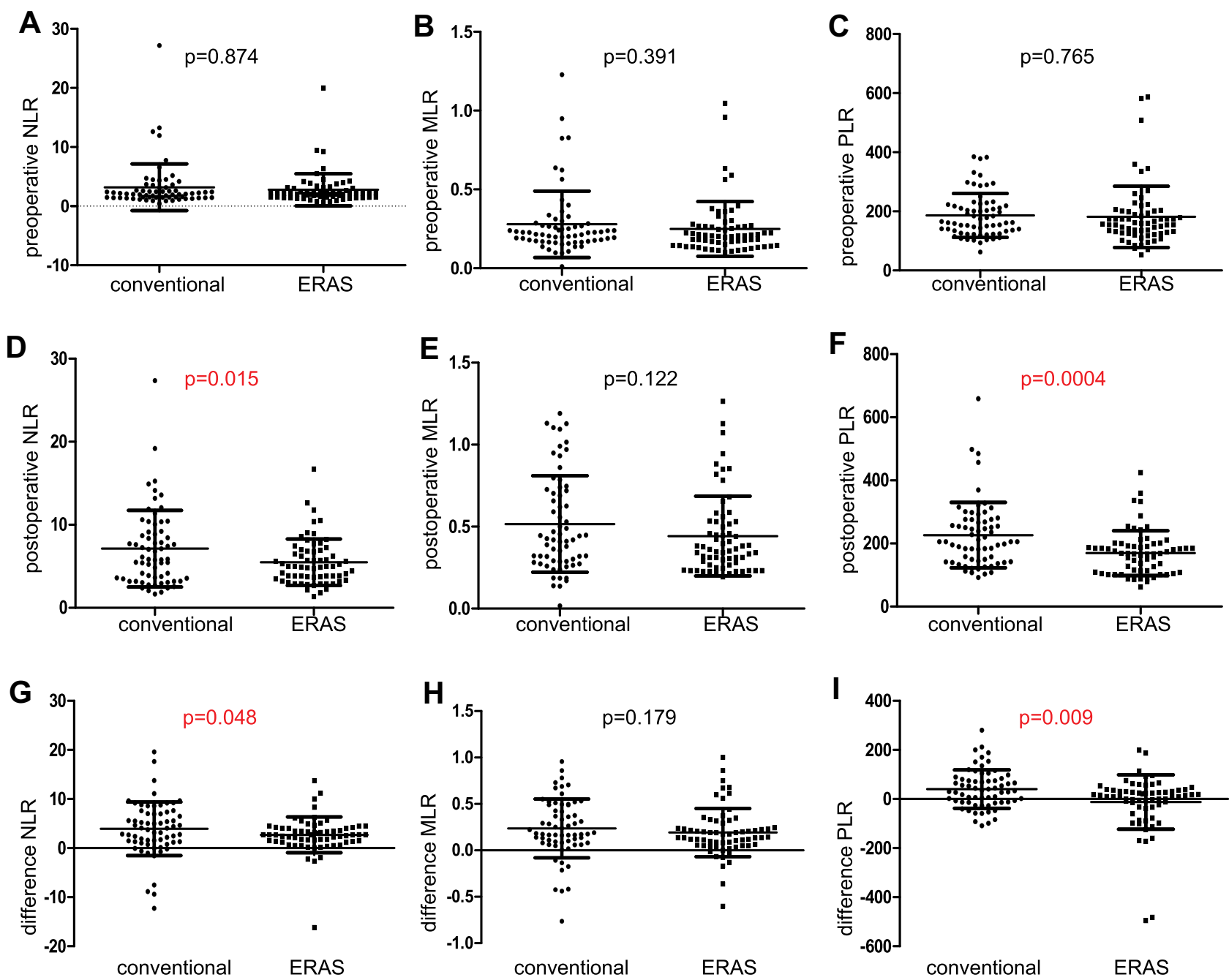

Figure 4 Comparison of patients with ERAS protocols and patients with conventional protocols, following gynecologic oncology surgery, in terms of preoperative NLR (A), MLR (B), and PLR (C); postoperative NLR (D), MLR (E), and PLR (F); difference between preoperative and postoperative NLR (G), MLR (H), and PLR (I).

The cellular response to surgical injury is to activate neutrophils and macrophages of the innate immune system by the production of proinflammatory cytokines, such as tumor necrosis factor (TNF) alpha, and the interleukins (ILs), for example, IL-1 and IL-6. Proinflammatory cytokines alter the levels of circulating acute-phase proteins such as C-reactive protein (CRP), albumin, ferritin, transferrin, and fibrinogen. ${ }^{33}$ It is reported that laparoscopic surgery and goal-directed fluid therapy can reduce postoperative CRP levels. ${ }^{34,35}$ Although laparoscopic surgery generates a reduced postoperative SIR following colorectal surgery, evidence of the effect of ERAS protocols on SIR to the gynecological oncology surgery is limited.

We here firstly screened the objective marker of SIR for ERAS following gynecological oncology surgery and identified that ERAS protocols decreased postoperative platelets,
PLR and NLR, which alleviated excessive inflammatory status in patients with gynecological malignancy. We demonstrated that ERAS protocols have a beneficial effect on postoperative SIRs, which could be the novel marker to estimate the implementation of an ERAS pathway for gynecologic oncology surgery. Recently, a great number of studies have shown that SIR is a predictor of prognosis in various cancers. ${ }^{36-38}$ Meanwhile, the objective marker of SIR has been explored as prognostic factors in ovarian cancer. Furthermore, many studies have reported that higher neutrophil or lower lymphocyte counts predict poorer survival in ovarian cancer. $^{23}$ Moreover, previous studies show a correlation between oncology survival and ERAS protocol. ${ }^{30}$ Therefore, SIR may be superior to length of stay for the evaluation of the success of ERAS, whereas our data show similar mortality; thus, the relationship between ERAS 
and survival in gynecological cancer can be analyzed if a longterm, follow-up study with enlarged sample size is designed. Otherwise, numerous facets of ERAS can be explored in future studies. The novelty of our study is that postoperative platelets, PLR or NLR could be available and inexpensive marker to estimate the success of ERAS protocols.

\section{Conclusion}

The ERAS protocol provides faster recovery rate, less postoperative pain and decreased incidence of PONV after gynecological oncology surgery without increasing complication or readmission rates. Furthermore, we present the implementation of a multimodal ERAS pathway for gynecological tumor surgeries that results in shorter hospital stay and lesser hospital costs. Otherwise, postoperative platelets, PLR or NLR may be utilized as a simple and easily obtained predictive marker for ERAS on gynecology oncology surgery. Future studies with multicenter patient collection are needed to determine which individual interventions from ERAS contribute the most to quality of recovery, as well as modulation of ERAS implementation on the novel SIR markers.

\section{Data Statement}

The databases used and/or analyzed during the current study are not publicly available (to maintain privacy) but can be available from the corresponding authors on reasonable request.

\section{Ethical Approval}

This study was approved by the Ethics Committee of Qilu Hospital (scientific research review N0.2018-141).

\section{Author Contributions}

All authors made a significant contribution to the work reported, whether that is in the conception, study design, execution, acquisition of data, analysis and interpretation, or in all these areas; took part in drafting, revising or critically reviewing the article; gave final approval for the version to be published; have agreed on the journal to which the article has been submitted; and agreed to be accountable for all aspects of the work.

\section{Funding}

This work was supported by grants from the National Natural Science Foundation of China (Project Nos. 81602286), and the Department of Medical and Health Science Technology of Shandong province (No. 2016w0345), and from the Department of Science
Technology of Jinan city (No. 201705051), and from the China Postdoctoral Science Foundation (Project Nos. 2019T120594) and Shandong natural science united foundation for cancer control (No. ZR2019LZL002).

\section{Disclosure}

The authors have no conflicts of interest to declare.

\section{References}

1. Gibb A, Crosby MA, McDiarmid C, et al. Creation of an Enhanced Recovery After Surgery (ERAS) Guideline for neonatal intestinal surgery patients: a knowledge synthesis and consensus generation approach and protocol study. BMJ Open. 2018;8(12):e023651. doi:10.1136/bmjopen-2018-023651

2. Gustafsson UO, Scott MJ, Hubner M, et al. Guidelines for perioperative care in elective colorectal surgery: enhanced recovery after surgery (ERAS ${ }^{\circledR}$ ) Society Recommendations: 2018. World J Surg. 2019;43(3):659-695.

3. Batchelor T, Rasburn NJ, Abdelnour-Berchtold E, et al. Guidelines for enhanced recovery after lung surgery: recommendations of the Enhanced Recovery After Surgery (ERAS ${ }^{\circledR}$ ) Society and the European Society of Thoracic Surgeons (ESTS). Eur $J$ Cardiothorac Surg. 2019;55(1):91-115.

4. Nelson G, Bakkum-Gamez J, Kalogera E, et al. Guidelines for perioperative care in gynecologic/oncology: enhanced Recovery After Surgery (ERAS) Society recommendations-2019 update. Int J Gynecol Cancer. 2019;29(4):651-668. doi:10.1136/ijgc-2019000356

5. Melloul E, Hübner M, Scott M, et al. Guidelines for perioperative care for liver surgery: enhanced Recovery After Surgery (ERAS) Society Recommendations. World J Surg. 2016;40(10):2425-2440. doi:10.1007/s00268-016-3700-1

6. Low DE, Allum W, De Manzoni G, et al. Guidelines for Perioperative Care in Esophagectomy: enhanced Recovery After Surgery $\left(\right.$ ERAS $\left.^{\circledR}\right)$ Society Recommendations. World J Surg. 2019;43(2):299-330.

7. Temple-Oberle C, Shea-Budgell MA, Tan M, et al. Consensus Review of Optimal Perioperative Care in Breast Reconstruction: enhanced Recovery after Surgery (ERAS) Society Recommendations. Plast Reconstr Surg. 2017;139(5):1056e-1071e. doi:10.1097/PRS.0000000000003242

8. Ljungqvist O, Scott M, Fearon KC. Enhanced recovery after surgery: a review. JAMA Surg. 2017;152(3):292-298. doi:10.1001/ jamasurg.2016.4952

9. Rogers LJ, Bleetman D, Messenger DE, et al. The impact of enhanced recovery after surgery (ERAS) protocol compliance on morbidity from resection for primary lung cancer. $J$ Thorac Cardiovasc Surg. 2018;155(4):1843-1852. doi:10.1016/j. jtcvs.2017.10.151

10. Bisch SP, Wells T, Gramlich L, et al. Enhanced Recovery After Surgery (ERAS) in gynecologic oncology: system-wide implementation and audit leads to improved value and patient outcomes. Gynecol Oncol. 2018;151(1):117-123. doi:10.1016/j.ygyno.2018.08.007

11. Parise P, Ferrari C, Cossu A, et al. Enhanced Recovery After Surgery (ERAS) pathway in esophagectomy: is a reasonable prediction of hospital stay possible. Ann Surg. 2019;270(1):77-83. doi:10.1097/ SLA.000000000002775

12. Arrick L, Mayson K, Hong T, Warnock G. Enhanced recovery after surgery in colorectal surgery: impact of protocol adherence on patient outcomes. J Clin Anesth. 2019;55:7-12. doi:10.1016/j. jclinane.2018.12.034 
13. Committee on Gynecologic Practice. ACOG Committee Opinion No. 750: perioperative pathways: enhanced recovery after surgery. Obstet Gynecol. 2018;132(3):e120-e130. doi:10.1097/ AOG.0000000000002818

14. Ferrari F, Forte S, Sbalzer N, et al. Validation of an enhanced recovery after surgery protocol in gynecologic surgery: an Italian randomized study. Am J Obstet Gynecol. 2020;223(4):543.e1-543. e14. doi:10.1016/j.ajog.2020.07.003

15. Gramlich LM, Sheppard CE, Wasylak T, et al. Implementation of Enhanced Recovery After Surgery: a strategy to transform surgical care across a health system. Implement Sci. 2017;12(1):67. doi:10.1186/s13012-017-0597-5

16. Deng Y, Zhu J, Liu Z, Huang M, Chang DW, Gu J. Elevated systemic inflammatory responses, factors associated with physical and mental quality of life, and prognosis of hepatocellular carcinoma. Aging (Albany NY). 2020;12(5):4357-4370. doi:10.18632/aging.102889

17. Dolan RD, McSorley ST, Horgan PG, Laird B, McMillan DC. The role of the systemic inflammatory response in predicting outcomes in patients with advanced inoperable cancer: systematic review and meta-analysis. Crit Rev Oncol Hematol. 2017;116:134-146. doi:10.1016/j.critrevonc.2017.06.002

18. Dolan RD, Lim J, McSorley ST, Horgan PG, McMillan DC. The role of the systemic inflammatory response in predicting outcomes in patients with operable cancer: systematic review and meta-analysis. Sci Rep. 2017;7(1):16717.

19. Templeton AJ, McNamara MG, Šeruga B, et al. Prognostic role of neutrophil-to-lymphocyte ratio in solid tumors: a systematic review and meta-analysis. J Natl Cancer Inst. 2014;106(6):dju124. doi:10.1093/jnci/dju124

20. Li B, Zhou P, Liu Y, et al. Platelet-to-lymphocyte ratio in advanced Cancer: review and meta-analysis. Clin Chim Acta. 2018;483:48-56. doi:10.1016/j.cca.2018.04.023

21. Yodying H, Matsuda A, Miyashita M, et al. Prognostic significance of neutrophil-to-lymphocyte ratio and platelet-to-lymphocyte ratio in oncologic outcomes of esophageal cancer: a systematic review and meta-analysis. Ann Surg Oncol. 2016;23(2):646-654. doi:10.1245/ s10434-015-4869-5

22. Haram A, Boland MR, Kelly ME, Bolger JC, Waldron RM, Kerin MJ. The prognostic value of neutrophil-to-lymphocyte ratio in colorectal cancer: a systematic review. J Surg Oncol. 2017;115 (4):470-479. doi:10.1002/jso.24523

23. Zhao Z, Zhao X, Lu J, Xue J, Liu P, Mao H. Prognostic roles of neutrophil to lymphocyte ratio and platelet to lymphocyte ratio in ovarian cancer: a meta-analysis of retrospective studies. Arch Gynecol Obstet. 2018;297(4):849-857. doi:10.1007/s00404-0184678-8

24. Diem S, Schmid S, Krapf M, et al. Neutrophil-to-Lymphocyte ratio (NLR) and Platelet-to-Lymphocyte ratio (PLR) as prognostic markers in patients with non-small cell lung cancer (NSCLC) treated with nivolumab. Lung Cancer. 2017;111:176-181. doi:10.1016/j. lungcan.2017.07.024

25. Dupré A, Malik HZ. Inflammation and cancer: what a surgical oncologist should know. Eur J Surg Oncol. 2018;44(5):566-570. doi:10.1016/j.ejso.2018.02.209
26. Lattanzi S, Brigo F, Trinka E, Cagnetti C, Di Napoli M, Silvestrini M. Neutrophil-to-lymphocyte ratio in acute cerebral hemorrhage: a system review. Transl Stroke Res. 2019;10(2):137-145. doi:10.1007/s12975-018-0649-4

27. Stawicki SP, Kalra S, Jones C, et al. Comorbidity polypharmacy score and its clinical utility: a pragmatic practitioner's perspective. J Emerg Trauma Shock. 2015;8(4):224-231. doi:10.4103/09742700.161658

28. Agha R, Abdall-Razak A, Crossley E, et al. STROCSS 2019 Guideline: strengthening the reporting of cohort studies in surgery. Int J Surg. 2019;72:156-165. doi:10.1016/j.ijsu.2019.11.002

29. Kalogera E, Bakkum-Gamez JN, Jankowski CJ, et al. Enhanced recovery in gynecologic surgery. Obstet Gynecol. 2013;122(2 Pt 1):319-328. doi:10.1097/AOG.0b013e31829aa780

30. Wijk L, Udumyan R, Pache B, et al. International validation of enhanced recovery after surgery society guidelines on enhanced recovery for gynecologic surgery. Am J Obstet Gynecol. 2019;221 (3):237.e1-237.e11. doi:10.1016/j.ajog.2019.04.028

31. McLeod RS, Aarts MA, Chung F, et al. Development of an enhanced recovery after surgery guideline and implementation strategy based on the knowledge-to-action cycle. Ann Surg. 2015;262 (6):1016-1025. doi:10.1097/SLA.0000000000001067

32. Aarts MA, Rotstein OD, Pearsall EA, et al. Postoperative ERAS interventions have the greatest impact on optimal recovery: experience with implementation of ERAS across multiple hospitals. Ann Surg. 2018;267(6):992-997. doi:10.1097/SLA.0000000000002632

33. Lord JM, Midwinter MJ, Chen YF, et al. The systemic immune response to trauma: an overview of pathophysiology and treatment. Lancet. 2014;384(9952):1455-1465. doi:10.1016/S0140-6736(14) 60687-5

34. Zang YF, Li FZ, Ji ZP, Ding YL. Application value of enhanced recovery after surgery for total laparoscopic uncut Roux-en-Y gastrojejunostomy after distal gastrectomy. World J Gastroenterol. 2018;24 (4):504-510. doi:10.3748/wjg.v24.i4.504

35. Funk DJ, HayGlass KT, Koulack J, Harding G, Boyd A, Brinkman R. A randomized controlled trial on the effects of goal-directed therapy on the inflammatory response open abdominal aortic aneurysm repair. Crit Care. 2015;19:247. doi:10.1186/s13054-015-0974-X

36. Ren K, Yin Y, He F, Shao Y, Wang S. Prognostic role of derived neutrophil-to-lymphocyte ratio in surgical triple-negative breast cancer. Cancer Manag Res. 2018;10:4891-4898. doi:10.2147/ CMAR.S180695

37. Tiainen S, Rilla K, Hämäläinen K, Oikari S, Auvinen P. The prognostic and predictive role of the neutrophil-to-lymphocyte ratio and the monocyte-to-lymphocyte ratio in early breast cancer, especially in the HER2+ subtype. Breast Cancer Res Treat. 2021;185(1):63-72. doi:10.1007/s10549-020-05925-7

38. Cong R, Kong F, Ma J, Li Q, Wu Q, Ma X. Combination of preoperative neutrophil-lymphocyte ratio, platelet-lymphocyte ratio and monocyte-lymphocyte ratio: a superior prognostic factor of endometrial cancer. BMC Cancer. 2020;20(1):464. doi:10.1186/s12885020-06953-8

\section{Publish your work in this journal}

Cancer Management and Research is an international, peer-reviewed open access journal focusing on cancer research and the optimal use of preventative and integrated treatment interventions to achieve improved outcomes, enhanced survival and quality of life for the cancer patient.
The manuscript management system is completely online and includes a very quick and fair peer-review system, which is all easy to use. Visit http://www.dovepress.com/testimonials.php to read real quotes from published authors. 